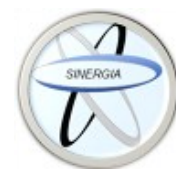

Revista Médica Sinergia

Vol. 7, Núm. 1, enero 2022, e751 https://doi.org/10.31434/rms.v7i1.751

revistamedicasinergia@gmail.com

\title{
Abdomen agudo: etiologías comunes en el embarazo
}

\section{Acute abdomen: common etiologies in pregnancy}

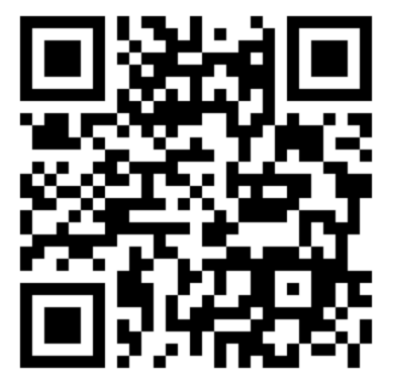

Recibido $17 / 10 / 2021$
Corregido $10 / 11 / 2021$

${ }^{1}$ Dra. Dayana Carvajal Camacho Hospital San Vicente de Paul, Heredia, Costa Rica

(i) https://orcid.org/0000-0002-1497-2033

${ }^{2}$ Dra. María José Durán Méndez Investigadora independiente, San José, Costa Rica

(D) https://orcid.org/0000-0001-6024-0864

${ }^{2}$ Dra. Marianela Sanchún Chacón Investigadora independiente, San José, Costa Rica

(i) https://orcid.org/0000-0002-2286-9909

\section{RESUMEN}

El abdomen agudo en el embarazo es una entidad clínica que se presenta en aproximadamente con una incidencia 2 de cada 1000 gestantes, su definición dice que es "un dolor abdominal súbito y agudo con una evolución rápida que requiere tratamiento urgente y puede llevar hasta la muerte en caso de no recibir tratamiento oportuno". Su progreso es rápido y sus síntomas se superponen con síntomas propios del embarazo.

Para la metodología se decidió realizar una revisión bibliográfica de diferentes artículos recientes para obtener la información más actualizada al momento de la elaboración de este documento.

Con ayuda de la recopilación de datos realizada en conjunto con el análisis de los mismos, al resumir en forma breve las diferencias clínicas y la metodología diagnóstica de elección para las causas más frecuentes, dentro de las recomendaciones están realizar una adecuada historia clínica completa dirigida, junto con una exploración física exhaustiva debido a la dificultad que existe en la examinación abdominal de una paciente en estado de embarazo, se puede elegir entre los diagnósticos diferenciales de las etiologías más comunes que se mencionarán en el artículo de manera resumida para realizar exámenes de laboratorio orientados y definir los casos donde es necesario la intervención quirúrgica por riesgo incrementado para una o ambas partes del binomio. 


\section{PALABRAS CLAVE: abdomen agudo; complicaciones; apendicitis; embarazo; desprendimiento de placenta.}

\section{ABSTRACT}

Acute abdomen in pregnancy is a clinical entity that occurs in approximately 2 in every 1000 pregnant women, its definition says that it is "sudden and acute abdominal pain with a rapid evolution that requires urgent treatment and can lead to death. in case of not receiving timely treatment". Her progress is rapid, and her symptoms overlap with symptoms typical of pregnancy.

For the methodology, it was decided to carry out a bibliographic review of different recent articles to obtain the most up-to-date information at the time of preparing this document.

With the help of the data collection carried out in conjunction with the analysis thereof, by briefly summarizing the clinical differences and the diagnostic methodology of choice for the most frequent causes, within the recommendations an adequate directed complete clinical history is made, together with a thorough physical examination due to the difficulty in abdominal examination of a pregnant patient, one can choose from the differential diagnoses of the most common etiologies mentioned in the article in a summarized way to perform laboratory tests oriented and define the cases where surgical intervention is necessary due to increased risk for one or both parts of the binomial.

KEYWORDS: acute abdomen; complications; appendicitis; pregnancy; placental abruption.

${ }^{1}$ Médica general, graduada de la Universidad de Ciencias Médicas (UCIMED). Cód. MED16616. Correo: dayanacam93@yahoo.es

${ }^{2}$ Médica general, graduada de la Universidad de Ciencias Médicas (UCIMED). Cód. MED16654. Correo: duranmendezmi@gmail.com

${ }^{3}$ Médica general, graduada de la Universidad de Ciencias Médicas (UCIMED). Cód. MED16655. Correo: nsanchun@gmail.com

\section{INTRODUCCIÓN}

El abdomen agudo en el embarazo es una entidad clínica que se presenta en aproximadamente 2 de cada 1000 gestantes y cuando se presenta usualmente requiere una resolución quirúrgica pronta, por lo que es de vital importancia conocer las principales etiologías y formas de presentación del cuadro para dar una resolución sin demora y disminuir la morbimortalidad materno-fetal asociada al mismo $(1,2)$.

Lo primero es conocer qué es el abdomen agudo, y su definición dice que es "un dolor abdominal súbito y agudo con una evolución rápida que requiere tratamiento urgente $\mathrm{y}$ puede llevar hasta la muerte en caso de no recibir tratamiento oportuno" (3).

El abdomen agudo puede ser tanto de resolución quirúrgica como no quirúrgica. En cuanto al abdomen agudo de tipo quirúrgico en embarazadas, las principales etiologías dependen del trimestre en que se encuentre la gestante, pero cabe recalcar que la apendicitis aguda es la causa más frecuente de las causas tanto obstétricas como no obstétricas.

El objetivo de esta revisión es repasar las etiologías comunes para poder reconocer de manera eficaz síntomas y signos, así como el diagnóstico y tratamiento de abdomen agudo en la gestación de origen no traumático, en forma breve. $(1,2)$ 
El presente trabajo se enfocó en recopilar información de diferentes artículos que abarcan la enfermedad de manera amplia, tratando así de resumir los conocimientos de forma práctica para el uso diario de un médico general.

\section{MÉTODO}

Se llevó a cabo una investigación científica por medio de una detallada revisión bibliográfica donde se examinaron, analizaron y sintetizaron diferentes artículos relacionados con las etiologías de abdomen agudo durante el embarazo para obtener en un solo documento un resumen práctico del abordaje de este tipo de pacientes.

Para recolectar y escoger los artículos se buscó en revistas médicas digitales asociadas a la Biblioteca Nacional de Salud y Seguridad Social, así como libros actuales, para realizar la presente revisión, con periodo de publicación desde 2016 para garantizar obtener los datos más actualizados, en ellas se hicieron búsquedas con las siguientes palabras clave: "abdomen agudo durante el embarazo", "manejo del abdomen agudo", "abdomen agudo quirúrgico en el embarazo", "dolor abdominal en embarazo tardío", "cirujano general y embarazo", "perforación intestinal en el embarazo", "imágenes no fetales durante abdomen agudo", "cirugía no obstétrica durante el embarazo", "torsión de ovario", "embarazo heterotópico" y "cirugía laparoscópica en abdomen agudo durante el embarazo"

Para el análisis de los datos se integraron conocimientos adquiridos durante la carrera y se resumió la información, en forma clara y precisa, de los diferentes artículos seleccionados.

\section{CAMBIOS FISIOLÓGICOS DURANTE LA GESTACIÓN}

Dentro de los cambios fisiológicos que conlleva el embarazo están el incremento del metabolismo del hígado, y debido a la hemodilución fisiológica la albúmina se ve reducida, y hay un descenso de las transaminasas. Sin embargo, la fosfatasa alcalina se incrementa debido a la producción de la placenta. Además, cabe recalcar que el embarazo por sí mismo, es un estado inflamatorio, por lo que los marcadores inflamatorios también se verán alterados. Otros cambios fisiológicos durante el embarazo se pueden ver en la tabla 1 (4).

La gestación es un periodo protrombótico y de tromboembolismo venoso, y su riesgo se ve aumentado por causa de la deshidratación, los procedimientos quirúrgicos y la sepsis (5). Para evitar fatalidades, toda mujer embarazada con abdomen agudo debe ser valorada con una historia clínica completa, pues ésta se correlaciona con la enfermedad y para ello funciona recordar la mnemotecnia ALICIA para recabar información sobre el dolor: Antigüedad y comienzo, Localización, Intensidad, Carácter, Irradiación, Atenuación 0 alivio (5).

En la exploración física, se inspecciona el nivel de hidratación, y otras anormalidades notorias, se palpan los cuatro cuadrantes abdominales y el útero grávido, este último dificulta la localización del dolor y podría enmascarar la intensidad del mismo, así como la peristalsis intestinal.

Se debe considerar el monitoreo fetal en todos estos casos, para asegurar el bienestar fetal. 


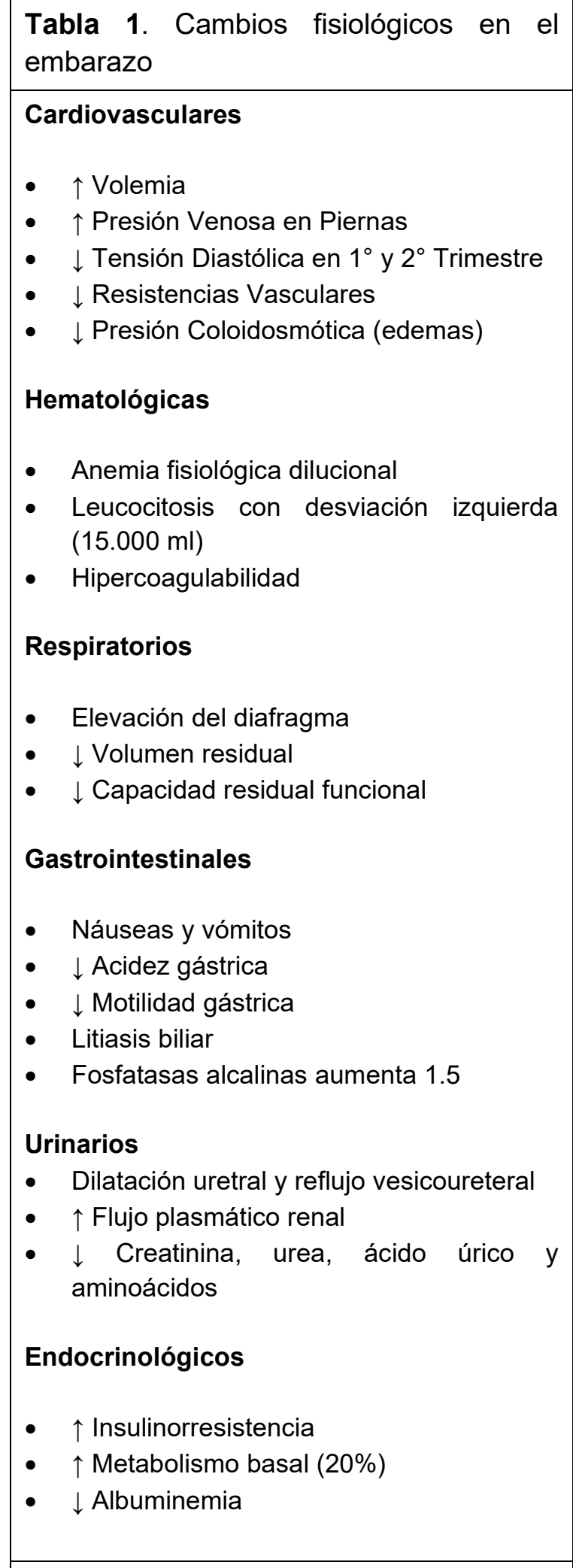

Fuente. Barber S, Bueno J, Granero P, Gómez I, Ballester N, García R. Actualización en el manejo del abdomen agudo no obstétrico en la paciente gestante. Elsevier.

\section{CAUSAS NO OBSTÉTRICAS DE ABDOMEN AGUDO EN GESTANTES}

Para el manejo de estas causas no obstétricas, se evitan las cirugías en el primer y tercer trimestre para evitar pérdidas del producto o una labor pretérmino respectivamente, sin embargo, si es una urgencia quirúrgica se debe proceder a la misma para evitar una fatalidad (6). Alrededor de $0.5 \%$ a $2.0 \%$ de todas las mujeres embarazadas con este tipo de abdomen agudo requerirá de una cirugía (711). A continuación, se describen causas no obstétricas de abdomen agudo:

\section{Apendicitis}

Esta es la patología más común de abdomen agudo que presenta la mujer embarazada sin importar el trimestre, con una incidencia de $1 / 1500$ embarazos $(7,11)$. Siendo más común en el segundo trimestre, y la perforación apendicular ha sido reportada con más frecuencia en el tercer trimestre (10).

Entre los síntomas que nos hacen sospechar de ella, se encuentran dolor abdominal que inicialmente es periumbilical o epigástrico que migra al cuadrante inferior derecho, náuseas, y vómito; cabe recordar que después de la semana 12 el aumento del útero grávido distorsiona el signo de protección y rebote que típicamente se pueden observar en una mujer no grávida y se distribuye este en toda el área del abdomen (5).

Entre los cambios de laboratorio, se observa una leucocitosis de probable causa fisiológica por el embarazo, y no significa que se tiene apendicitis aguda, sin embargo, si esta leucocitosis se acompaña de desviación a la izquierda con presencia de bandas, nos orienta a una patología de 
origen inflamatorio. $Y$ se puede confirmar por medio de ultrasonido (7).

La apendicitis aguda en la mujer embarazada es una patología que debe excluirse y no confundirse con las molestias que se presentan frecuentemente en los últimos meses de la gestación, por los propios cambios fisiológicos de la gravidez.

El tratamiento definitivo se realiza por medio de cirugía (8-10).

\section{$\underline{\text { Colecistitis }}$}

Siguiendo el orden de frecuencia se encuentra la colecistitis, con una incidencia muy amplia desde 1/1600 a 1/10.000 embarazadas. Se ha reportado litiasis biliar asintomática en alrededor de $3.5 \%$ de las embarazadas y el $90 \%$ de las colecistitis sufre predisposición para padecer colelitiasis $(10,11)$.

Entre los factores predisponentes, además de ser mujer gestante, están: alto índice de masa corporal, inactividad física, historia de desórdenes digestivos e historia familiar.

La presentación clínica es similar a la de la mujer no embarazada: presentándose dolor de inicio súbito que persiste 3 horas postprandial. El dolor descrito es de tipo cólico, desde leve a severo, o constante en cuadrante superior derecho o en epigastrio, y se asocia a náuseas, anorexia, vómitos, y fiebre $(10,11)$.

Las pruebas de laboratorio que orientan al diagnóstico son leucocitosis con presencia de bandemia, elevación de las enzimas hepáticas, aumento de la bilirrubina directa. El ultrasonido es la prueba de imagen de elección por su alta sensibilidad, pues detecta litos de hasta $2 \mathrm{~mm}$ de diámetro (7). Se prefiere la cirugía laparoscópica para la colecistectomía, ante el tratamiento médico, puesto que el riesgo de muerte fetal disminuye de $7 \%$ a $2.2 \%$. $Y$ en caso de complicaciones se recomienda la colangiopancreatografía

retrógrada endoscópica (CPRE) $(9,10)$.

\section{Obstrucción Intestinal}

Esta patología es extremadamente rara en el embarazo con incidencia de 1/3.000 embarazos, sin embargo, tiene alta tasa de pérdidas fetales $(17 \%)$ y muerte materna (2\%). Se presentan con mayor frecuencia en embarazos avanzados, con dos picos mayor índice de incidencia, el primero de la semana 16 a la 20 , y el segundo desde la semana 36 hasta el puerperio inmediato.

La causa más común son las adherencias, vólvulos, intususcepción, y hernias (11).

La sintomatología presente incluye náusea, vómito, dolor abdominal, distensión abdominal y constipación absoluta (11).

En la evaluación clínica se dificulta la distensión por el abdomen grávido, con posibles sonidos intestinales de hiperperistaltismo que se tornan hipoperistálticos lo cual traduce en estrangulación intestinal (10).

Las radiografías abdominales dan información valiosa ante la sospecha clínica (7).

El tratamiento inicial es conservador: descanso intestinal, hidratación intravenosa, y aspiración nasogástrica con monitoreo cercano. En caso de que este falle, se vuelve obligatoria una intervención quirúrgica urgente por medio de laparotomía (6,9-12).

\section{Pancreatitis}

Su incidencia se reporta en $1 / 3.000$ embarazos, con mayor frecuencia en el tercer trimestre (11). Presenta una mortalidad materna de menos de $1 \%$, y también genera parto pretérmino en aproximadamente $20 \%$ (10).

La mayoría de las pancreatitis agudas son autolimitadas, sin embargo, pueden 
progresar a una condición severa hasta llegar a falla multiorgánica, shock e incluso la muerte $(10,11)$.

Las causas más frecuentes son secundarias a colelitiasis $(70 \%)$ e hipertrigliceridemia congénita $o$ adquirida.

La presentación se asemeja a adultas no embarazadas: náuseas de inicio súbito, vómito y dolor abdominal que irradia a espalda, que a veces alivia al inclinarse hacia adelante. Para su diagnóstico se necesitan valores elevados de amilasa y lipasa pancreáticas, y las pruebas de imagen no son tan útiles en estos casos $(6,7)$.

El tratamiento conservador es preferido: descanso intestinal, aspiración nasogástrica, adecuada hidratación, corrección de electrolitos y analgésicos (de preferencia meperidina), con mejoría de la mayoría de las pacientes al quinto día del inicio del tratamiento $(9,10)$.

\section{CAUSAS OBSTÉTRICAS DE ABDOMEN AGUDO}

Dentro de estas, podemos encontrar las de causa fisiológica, como el dolor que causa el ligamento redondo (10 a 30\% de embarazos al final del primer trimestre y principios del segundo), este dolor se localiza en los cuadrantes abdominales inferiores hasta la ingle, es de tipo cólico, que se incrementa con el movimiento y es más frecuente en multíparas. Por otro lado, entre las causas patológicas más frecuentes están el desprendimiento de placenta, el fibroma degenerativo y la ruptura uterina. Se incluye en la descripción el embarazo heterotópico por su aumento en los últimos años $(10,13)$.

\section{Desprendimiento de placenta}

El desprendimiento de la placenta normoinserta se le conoce como abruptio
Placentae con una incidencia de $0.6 \%$. Se puede sospechar clínicamente cuando hay sangrado vaginal con dolor abdominal en periodo anteparto. Sin embargo, se puede presentar sin sangrado, lo cual retrasa su diagnóstico. A la exploración física, se puede observar un abdomen más grande para la edad gestacional, aumento de la tensión abdominal e hipersensibilidad, la frecuencia cardiaca fetal puede evidenciar distrés fetal severo 0 incluso ausencia de ruidos cardiacos.

Ante esta situación se busca la vía de parto más rápida, con la ruptura artificial de membranas para reducir la liberación de tromboplastina (10).

\section{Torsión de anexos}

La torsión de anexos es más frecuente en el embarazo (28\%), que en mujeres no gestantes (7\%) (11). Esta condición no amenaza la vida de la paciente, pero sí es muy dolorosa al girar más de $40 \%$ en rotación axial. Es la etiología más frecuentemente asociada al síndrome de hiperestimulación durante una fertilización in vitro.

Se presenta inicialmente hipersensibilidad en el abdomen bajo, otros síntomas son náuseas, vómito, diarrea, constipación, fiebre leve y taquicardia, luego puede presentar dolor de más intensidad, shock y malestar intestinal y urinario. Además de shock vasovagal materno y asfixia fetal (10). Los métodos estándar que orientan al diagnóstico son el examen clínico, ultrasonido, laparoscopia, y hallazgos de laboratorio. En el ultrasonido se puede observar una masa anexial y con el doppler se documenta la presencia o ausencia de flujo sanguíneo $(6,7,11)$.

Si se sospecha de esta patología, se debe realizar cirugía sin retrasos, y el diagnóstico 
usualmente se realiza con la visualización directa durante la cirugía, donde se encuentra un ovario o trompa uterina rotada de color azulada o negruzca. Se deben destorsionar los ovarios y preferiblemente realizar una cistectomía. En caso de masas anexiales asintomáticas de $5 \mathrm{~cm}$ de diámetro descubiertas en el primer trimestre, es común que resuelvan en forma espontánea en un $70-85 \%$ de las veces, por tanto, se prefiere el manejo conservador en estos casos $(11,14)$.

\section{Fibroma uterino}

Los fibroides son diagnosticados incidentalmente durante los ultrasonidos de rutina obstétricos. Muchos de ellos son asintomáticos. Sin embargo, entre el 5 - 15\% producen dolor intenso debido a que la degeneración del fibroide supera su suministro sanguíneo.

Los síntomas y signos son muy inespecíficos junto con dolor abdominal bajo.

En la examinación física se revela hipersensibilidad sobre el área del fibroide, por lo cual su diagnóstico es por medio de ultrasonido. Su tratamiento es conservador con administración corta de indometacina así que muy rara vez se requiere resolución quirúrgica $(7,11,13)$.

\section{$\underline{\text { Ruptura uterina }}$}

La incidencia de esta enfermedad ha venido en disminución y varía entre 1/10.000 a $1 / 15.000$ nacimientos. Pero se presenta con frecuencia de 0.5 a $1 \%$ en cicatrices uterinas previas. Este descenso se debe a que la multiparidad ha venido en descenso y a un uso racional de la oxitocina y la prostaglandina (11). La ruptura uterina puede ser primaria: en un útero intacto, o secundaria: en caso de presentarse una cicatriz previa por una cirugía o daño uterino por un útero anómalo.

La presentación clínica es de dolor abdominal severo de inicio súbito con signos peritoneales, ausencia de ruidos cardiacos fetales, ausencia de contracciones uterinas, sangrado vaginal y shock, no todos los síntomas tienen que estar necesariamente presentes. También el monitoreo cardiotocográfico puede mostrar desaceleraciones variables seguidas de bradicardia. Si fuera una ruptura uterina franca, ésta causaría muerte fetal, hemoperitoneo, pérdida de la tonicidad del útero, sangrado vaginal, relieve de las partes fetales a través del abdomen y posible hematuria.

Se necesita un ultrasonido urgente en sala de labor para confirmar el diagnóstico seguida de una laparotomía inmediata con resucitación materna, parto y reparación uterina, o histerectomía en caso de no poder realizarse la reparación $(7,10,11)$.

\section{Embarazo heterotópico}

Esta patología posee una prevalencia baja, pero que en los últimos años ha ido en aumento debido a las técnicas de reproducción asistida, se estima que puede llegar a ser casi de $1 \%$ en algunas series. La incidencia de supervivencia del producto intrauterino es de alrededor $60-70 \%$. El diagnóstico en su gran mayoría se hace en el primer trimestre de gestación.

Dentro de los factores de riesgo, tanto para el embarazo ectópico como para el heterotópico, se incluyen: embarazo ectópico previo, cirugía tubárica o pélvica previa, técnicas de reproducción asistida, adherencias relacionadas con la endometriosis o no y la enfermedad pélvica inflamatoria. 
Las características clínicas pueden variar ampliamente desde asintomático (cuando no se ha roto el embarazo ectópico), dolor abdominal, choque hipovolémico con dolor abdominal, con o sin una masa anexial, irritación peritoneal, sangrado vaginal hasta síntomas atípicos como dolor en epigastrio y vómitos. Se debe sospechar de esta patología en toda paciente con amenorrea, dolor abdominal, irritación peritoneal y útero agrandado, aún incluso si posee un embarazo intrauterino confirmado, puesto que el diagnóstico temprano disminuye la morbimortalidad materna y preserva su función reproductiva.

El procedimiento quirúrgico laparoscópico ha demostrado ser el mejor método para el diagnóstico y tratamiento de esta patología, ya que el ultrasonido transvaginal posee una sensibilidad de $40 \%$ y el abdominal hasta un $50 \%$.

El tratamiento conservador es medicamentoso y se prefiere en caso de no contar con un embarazo intrauterino $(6,7,13,15)$.

\section{ANÁLISIS}

Con la recopilación de datos realizada en conjunto con el análisis de los mismos, se lograron simplificar las diferencias clínicas y la metodología diagnóstica de elección para las causas más frecuentes de abdomen agudo durante la gestación, siendo así la apendicitis aguda la de mayor incidencia, sin importar el trimestre en el que se encuentre la gestante, diferenciándose de la segunda causa con la colecistitis por medio del tiempo y localización inicial del dolor, en apendicitis es de inicio súbito periumbilical que luego migra a cuadrante inferior derecho, y en colecistitis es posprandial en cuadrante superior derecho, apoyándose en ambas con el ultrasonido para definir el diagnóstico.
La obstrucción intestinal y la pancreatitis son de menor incidencia, siendo la radiografía una opción diagnóstica de bajo costo para la primera y la pancreatitis se diagnóstica por descarte entre las no obstétricas, ya que podemos llegar al mismo con exámenes de laboratorio que se realizan con frecuencia en el abordaje inicial, del estudio de un dolor abdominal.

Por otro lado, el desprendimiento de placenta es la causa más común de abdomen agudo obstétrico, que comparte con la ruptura uterina el signo de sangrado vaginal, y la necesidad de un abordaje materno-fetal con parto rápido. La ruptura presenta historia de una cicatriz uterina por cirugía previa, y requiere laparotomía exploratoria inicial en todos los casos.

Tanto para la torsión anexial y el embarazo heterotópico el diagnóstico y su tratamiento pueden realizarse con una laparoscopia, en el caso del fibroma uterino al ser la mayoría asintomático y de baja incidencia, su terapia es conservadora.

Es importante recalcar que en todas ellas hay hipersensibilidad abdominal baja y de no ser tratadas llevan a inestabilidad hemodinámica del binomio madre-hijo.

\section{CONCLUSIONES}

Es fundamental que el médico-clínico conozca con total claridad los cambios fisiológicos de cada trimestre para que logre diferenciar las molestias gastrointestinales del dolor abdominal patológico.

Dentro de las recomendaciones están realizar una adecuada historia clínica completa dirigida con antecedentes ginecoobstétricos y quirúrgicos, junto con un examen físico exhaustivo debido a la dificultad que existe por el embarazo. Cuando se defina la ubicación principal de dolor y tiempo de evolución del mismo, se 
puede elegir de manera más precisa, entre los diagnósticos diferenciales de las etiologías más comunes mencionadas en el artículo para realizar exámenes de laboratorio orientados en el abordaje inicial de un dolor abdominal, teniendo en cuenta que dichos laboratorios como la leucocitosis puede estar enmascarada por fisiología normal del embarazo, al igual que se alteran las pruebas de función hepática y otros exámenes utilizados con frecuencia. Reservando el ultrasonido y la laparoscopia exploratoria como herramientas, cuando no está tan claro el diagnóstico a través de la clínica.

Además, se aprovecha la laparoscopia como tratamiento en varias causas tanto obstétricas y no obstétricas de abdomen agudo, en casos donde la anterior no esté indicada se realizará la intervención por laparotomía como siguiente opción terapéutica, en los casos donde el riesgo para una o ambas partes del binomio esté incrementado.

Sin embargo, se prefiere el tratamiento conservador hasta cuando la situación lo permita.

\section{Los autores declaran no tener conflicto de interés.}

\section{REFERENCIAS}

1. Rosendorf J, Liška V, Pálek R, Třeška V. Acute abdomen in pregnancy: a retrospective study of pregnant patients hospitalised for abdominal pain. proLékařecz [Internet]. 2020 [cited 26 February 2021];99(3):131-135. Available from: https://www.prolekare.cz/en/journals/perspectives -in-surgery/2020-3-2/acute-abdomen-inpregnancy-a-retrospective-study-of-pregnantpatients-hospitalised-for-abdominal-pain-122065

2. Laffita W, Rodríguez I, Jiménez W, Jeremías E. Abdomen agudo quirúrgico en el embarazo. Revista Información Científico [Internet]. 2018 [cited 16 January 2021];Volumen 97(No. 6):10761087.

Available from: http://scielo.sld.cu/scielo.php?script=sci arttext\& pid=S1028-99332018000601076

3. 2017. Tratado de Cirugía General. 3rd ed. Ciudad de México: El Manual Moderno, p.274.

4. Barber S, Bueno J, Granero P, Gómez I, Ballester N, García R. Actualización en el manejo del abdomen agudo no obstétrico en la paciente gestante. elsevier [Internet]. 2016 [cited 26 February 2021];94(No.5):257-265. Available from: https://www.elsevier.es/en-revista-cirugiaespanola-36-articulo-actualizacion-el-manejo-delabdomen-S0009739X15003097

5. Chilaka C, F Walker K. Abdominal pain in late pregnancy. Obstetrics, Gynaecology and Reproductive Medicine [Internet]. 2019 [cited 14 January 2021];29(12):331-341. Available from: https://www.obstetrics-gynaecologyjournal.com/article/S1751-7214(19)30172-1/pdf

6. Vujic J, Marsoner K, Lipp-Pump A, Klaritsch P, Mischinger $\mathrm{H}$, Kornprat $\mathrm{P}$. Non-obstetric surgery during pregnancy - an eleven-year retrospective analysis. BMC Pregnancy and Childbirth [Internet]. 2019 [cited 16 January 2021];19(382):15. Available from: https://bmcpregnancychildbirth.biomedcentral.co m/track/pdf/10.1186/s12884-019-2554-6.pdf

7. C. Moreno C, K. Mittal P, H. Miller F. Nonfetal Imaging During Pregnancy Acute Abdomen/Pelvis. Radiologic Clinics is published by Elsevier [Internet]. 2020 [cited 15 January 2021];58(2):363-380. Available from: https://linkinghub.elsevier.com/retrieve/pii/S00338389(19)30134-4

8. BRĂILA A, ZAVATE A, STEPAN A, SABETAY C, KAMEL J, GEORGESCU-BRĂILA $M$ et al. The acute abdomen in pregnancy and postpartum of a teenager woman. Case report. RJME Romanian Journal of Morphology \& Embryology [Internet]. 2018 [cited 15 January 2021];59(3):939 - 943. Available from: https://rime.ro/RJME/resources/files/5903189399 43.pdf

9. Rege S, Roshan C, Siddhant V, Shrinivas S, Ajinkya R. Laparoscopic surgery for non-obstetric acute abdomen in pregnancy: $A$ retrospective case series review. Journal of Minimal Access Surgery [Internet]. 2019 [cited 16 January 2021];16(1):54-58. Available from: https://www.ncbi.nlm.nih.gov/pmc/articles/PMC69 45339/

10. Koshy S, Fenn M, Jacob K, Alias S, Anna S. Management of acute abdomen in pregnancy: current perspectives. Int J Womens Health [Internet]. 2019 [cited 26 February 2021];11:119- 
134. Available from:

https://www.ncbi.nlm.nih.gov/pmc/articles/PMC63

71947/

11. Parikh B, Hussain F, Brustman L. Acute Abdomen in Pregnancy. Topics in Obstetrics \& Gynecology [Internet]. 2019 [cited 9 February 2021];39(7):1-7. Available from: https://insights.ovid.com/topicsobstetrics-gynecology/togy/2019/05/150/acuteabdomen-pregnancy/1/01938899

12. Kapadia S, Crabtree M, French S, DeUgarte D. In utero ileal volvulus and intestinal perforation associated with enteric duplication cyst presenting with preterm labour and acute abdomen in newborn. BMJ Journals [Internet]. 2019 [cited 15 January 2021];12(11):1-3. Available from: https://casereports.bmj.com/content/12/11/e2320 52.long

13. Hecht $S$, Meissnitzer M, Forstner R. Akutes Abdomen der Frau: gynäkologische Ursachen. SpringerLink [Internet]. 2018 [cited 26 February 2021];59(2):126-132. Available from: https://link.springer.com/article/10.1007/s00117018-0475-4

14. Habek D, Bauman R, Kralj R, Hafner T, Turudic T, Vujisic S. Acute Abdomen in the 17th Week of Twin Pregnancy due to Ovarian Torsion - A Late Complication of IVF. Geburtshilfe Frauenheilkd [Internet]. 2016 [cited 26 February 2021];76(12):1345-1349. Available from: https://www.ncbi.nlm.nih.gov/pmc/articles/PMC51 77560/

15. Hernández J, Tovar A, Noriega V, Izaguirre J, Moreno E, Valderrama O. El cirujano general frente al embarazo heterotópico roto. Presentación de un caso y revisión de la literatura. SciELO [Internet]. 2018 [cited 15 January 2021];40(1):24-30. Available from: http://www.scielo.org.mx/scielo.php?script=sci ar ttext\&pid=S1405-00992018000100024 